\title{
RINGS WHOSE CLASS OF PROJECTIVE MODULES IS
} SOCLE FINE

\author{
Abdelouahab Idelhadj, El Amin Kaidi, Dolores Martín \\ Barquero and Cándido Martín GonzÁlez
}

\begin{abstract}
A class $\mathcal{C}$ of modules over a unitary ring is said to be socle fine if whenever $M, N \in \mathcal{C}$ with $\operatorname{Soc}(M) \cong \operatorname{Soc}(N)$ then $M \cong N$. In this work we characterize certain types of rings by requiring a suitable class of its modules to be socle fine. Then we study socle fine classes of quasi-injective, quasi-projective and quasicontinuous modules which we apply to find socle fine classes in special types of noetherian rings. We also initiate the study of those rings whose class of projective modules is socle fine.
\end{abstract}

\section{Introduction}

The notion of socle fine class of modules has been previously used in the algebraic literature without an explicit formulation. Thus in $[\mathbf{5}$, Theorem 9.3 .7$, p. 166] it is proved that an algebra $A$ is quasi-Frobenius if and only if each principal $A$-module has a simple socle and, for any two nonisomorphic principal $A$-modules $P_{1}$ and $P_{2}$, we have $\operatorname{Soc}\left(P_{1}\right) \nsucceq \operatorname{Soc}\left(P_{2}\right)$. Clearly, this last assertion is equivalent to the fact that the class of principal A-modules is socle fine. Also, the result given in [5, Corollary 9.4.3, p. 171] could be stated by proclaiming that any class of indecomposable modules of the same finite length, over a uniserial algebra, is socle fine. Other results in this vein appear in [15] where some socle fine classes are found in the context of CEP-rings. More recently, Page and Zhou in [23, Theorem 24, p. 2920] find a series of equivalent conditions characterizing the socle fine character of certain natural classes. An explicit formulation of the notion was given by A. Idelhadj and E. A. Kaidi in [11] where they

2000 Mathematics Subject Classification. Primary: 13C13; Secondary: 16D10.

Key words. Socle fine class, projective module, QI-module, QP-module.

Supported by the Spanish DGICYT with project numbers BFM 2001-2335 and BFM 2001-1886, by the Junta de Andalucía projects: FQM-336, FQM 0194, and 'Estudio analítico-algebraico de Sistemas Triples y de Pares en diferentes clases de estructuras no asociativas'. 
give a socle fine characterization of semiartinian rings. Continuing this philosophy, artinian and noetherian rings are characterized in $[\mathbf{1 6}]$ by socle fine classes. The interesting paper [12] contains also characterizations for $V$-rings and pseudo-Frobenius rings. Complementary literature on socle and radical fine classes can be found in [13], [17] and [10].

Throughout this work the word ring will mean a unitary (associative) ring and modules are understood as unitary (left) modules. If $R$ is a ring and $X$ a class or $R$-modules, we shall say that $X$ is socle fine (respectively radical fine) whenever for any $M, N \in X$ we have $\operatorname{Soc}(M) \cong \operatorname{Soc}(N)$ $($ respectively $M / \operatorname{Rad}(M) \cong N / \operatorname{Rad}(N))$ if and only if $M \cong N$. We shall denote the injective hull of a module $M$ by $E(M)$.

\section{QI, QP and quasi-continuous modules}

Our main references for quasi-injective (QI), quasi-projective (QP) and quasi-continuous modules are $[\mathbf{2 2}]$ and $[\mathbf{1}]$. By introducing QI or QP modules into the scene, we obtain another characterization for semisimple rings:

Theorem 2.1. For any ring $R$, the following assertions are equivalent:

1) $R$ is semisimple.

2) The class of all QP modules is socle fine.

3) The class of all QI modules is socle fine.

4) The class $\mathcal{C}$ of all the finite direct sums of QP modules is socle fine.

Proof: If $R$ is semisimple the class of all $R$-modules is socle fine hence 1) implies 2), 3) and 4). Suppose 2). As $R$ and $\operatorname{Soc}(R)$ are QP and $\operatorname{Soc}(R)=\operatorname{Soc}(\operatorname{Soc}(R))$ we have $R \cong \operatorname{Soc}(R)$ implying that $R$ is semisimple. If 3) holds, since $\operatorname{Soc}(E(R))=\operatorname{Soc}(R)=\operatorname{Soc}(\operatorname{Soc}(R))$ and both of $E(R)$ and $\operatorname{Soc}(R)$ are QI, we have $E(R) \cong \operatorname{Soc}(R)$ is semisimple and so $R$ is also. Finally suppose 4 ). We shall prove that $R$ is semisimple by showing that the class of the QP $R$-modules is closed under finite direct sums. Let $X_{1}, \ldots, X_{n}$ be QP $R$-modules, and $X=\oplus_{i} X_{i}$ be an element in $\mathcal{C}$, let $Y:=\operatorname{Soc}(X)$ which is QP by its semisimple character. Since $X, Y \in \mathcal{C}$ and $\operatorname{Soc}(X)=\operatorname{Soc}(Y)$, we conclude $X \cong Y$ hence $X$ is semisimple and therefore QP. Now the fact that the class of QP modules is closed under finite direct sum implies that $R$ is semisimple (see [7] and $[\mathbf{2 0}])$.

Let $\mathcal{C}$ be a socle fine class, $M$ an element in $\mathcal{C}$ and $[M]$ the isomorphism class of $M$. Then the class $\mathcal{C} \cup[M]$ is also socle fine. Moreover if $\left\{M_{i}\right\}_{i \in I}$ is a collection of elements in $\mathcal{C}$, the class of $R$-modules $\mathcal{C} \cup\left(\cup_{i \in I}\left[M_{i}\right]\right)$ is 
socle fine. It is obvious that if $\mathcal{C}_{1}$ and $\mathcal{C}_{2}$ are two $R$-module socle fine classes such that $\forall X \in \mathcal{C}_{1}, \forall Y \in \mathcal{C}_{2}$, $\operatorname{Soc}(X) \neq \operatorname{Soc}(Y)$ then $\mathcal{C}_{1} \cup \mathcal{C}_{2}$ is a socle fine class.

Theorem 2.2. Let $M$ be a quasi-continuous $R$-module and $\mathcal{C}$ the class of its direct summands. Then $\mathcal{C}$ is socle fine if and only if $\operatorname{Soc}(M)$ is essential in $M$.

Proof: Let us suppose that $\mathcal{C}$ is socle fine. As $E(M)=E(\operatorname{Soc}(M)) \oplus W$ with $\operatorname{Soc}(W)=0$ and $M$ is quasi-continuous, by [22, Theorem 2.8, (4), p. 20] we have that $M=(M \cap E(\operatorname{Soc}(M))) \oplus(M \cap W)$, where $\operatorname{Soc}(M)$ is essential in $M \cap E(\operatorname{Soc}(M))$ and $\operatorname{Soc}(M) \cap W=0$. Since $M \cap W$ is a direct summand of $M$ with zero socle and $\mathcal{C}$ is socle fine, $M \cap W=0$ and $\operatorname{Soc}(M)$ is essential in $M$. Let us suppose now that $\operatorname{Soc}(M)$ is essential in $M$. Let $N$ be a direct summand of $M$. Then $\operatorname{Soc}(N)$ is essential in $N$ hence $E(N)=E(\operatorname{Soc}(N))$. Therefore if $N_{1}, N_{2} \in \mathcal{C}$, with $\operatorname{Soc}\left(N_{1}\right) \cong \operatorname{Soc}\left(N_{2}\right)$ we have that $E\left(N_{1}\right) \cong E\left(N_{2}\right)$ and by [22, Theorem 2.31, p. 34], we have $N_{1} \cong N_{2}$.

Corollary 2.1. Let $R$ be a ring. Then $R$ is a $V$-ring if and only if the class $C$ of its indecomposable QI modules with essential socle is socle fine. The ring $R$ is a noetherian $V$-ring if and only if the class $D$ of its $Q I$ modules with essential socle is socle fine.

Proof: Let $R$ be a $V$-ring and $M$ be an indecomposable QI $R$-module. Then $E(M)$ is also indecomposable (see [22, Theorem 2.8, p. 20]). Let $S$ be a simple (hence injective) submodule of $M$. Then $E(M)=S$ and so $M=S$. By the previous corollary, the class $C$ is socle fine. Reciprocally, if $C$ is socle fine take a simple $R$-module $S$. Then $E(S)$ is indecomposable and therefore $S, E(S) \in C$. Since $\operatorname{Soc}(S)=\operatorname{Soc}(E(S))$ we have $S \cong E(S)$ and so $S$ is injective. Thus $R$ is a $V$-ring.

Suppose now that $R$ is a noetherian $V$-ring. It is easy to prove that the indecomposable QI modules are semisimple hence they form a socle fine class $D$. On the other hand, if $D$ is socle fine, by the proved previous part of this corollary $R$ is a $V$-ring. Next we prove that any semisimple module is injective: let $M=\oplus_{i} S_{i}$ with each $S_{i}$ a simple submodule. Then $M \in D$ and as $\operatorname{Soc}(E(M))=\operatorname{Soc}(M)=M$ we deduce that $E(M)$ has an essential socle; then $M, E(M) \in D$ and $\operatorname{Soc}(M)=\operatorname{Soc}(E(M))$ hence $M \cong E(M)$ and so any semisimple module is injective. This implies that the class in [16, Theorem 3] is socle fine and therefore $R$ is noetherian. 
Corollary 2.2. Let $D$ be a noetherian domain with Krull dimension 1 and $\mathcal{C}$ any class of pairwise relatively injective torsion D-modules. Then $\mathcal{C}$ is socle fine.

Proof: By Theorem 2.2 it suffices to prove that any element $M \in \mathcal{C}$ has essential socle. Since $M$ is quasi-injective, each submodule of $M$ is essential in a summand of $M$ (see [22, Proposition 2.1, p. 18]). Then $M=T \oplus$ $W$ where $\operatorname{Soc}(M)$ is essential in $T$ (and $\operatorname{Soc}(W)=0$ ). As $E(W)$ is a direct sum of indecomposable injective $D$-modules, applying the corollary of [26, Theorem 2.32, p. 53] each injective indecomposable module is of the form $E(D / P)$ with $P$ a prime ideal of $D$. As $\operatorname{Soc}(W)=\operatorname{Soc}(E(W))=0$. Then each indecomposable component $E(D / P)$ of $E(W)$ has zero socle hence $P$ is not maximal, and so, since the Krull dimesion of $D$ is one, $P=0$. Consequently $E(W)=\oplus_{i \in I} Q_{i}$, with $Q_{i}=Q(D)$ the field of fractions of $D$. Then $T(E(W))=0$ implying $T(W)=0$. Furthermore $M=T(M)=T(T) \oplus T(W)=T(T) \subset T \subset M$, hence $M=T, W=0$ and $\operatorname{Soc}(M)$ is essential in $M$.

Corollary 2.3. If $D$ is a Dedekind domain, the class of the indecomposable D-modules of the same (finite) length is socle fine.

Proof: This corollary is a consequence of the structure theory of quasiinjective modules over Dedekind domains. We recall that, in this context, any QI module is either injective or a torsion $D$-module $M$ such that for each nonzero prime ideal $P$, the $P$-primary component $M_{P}$ of $M$ is a direct sum of isomorphic modules each one of them being isomorphic to $D / P^{n}(n>0)$ or to $E(D / P)$ (see $\left.[\mathbf{9}]\right)$. The indecomposable $D$-modules of finite length are torsion modules (see [21, Theorem 1, p. 49]), by the structure theory for finitely generated modules over a Dedekind domain, they have the form $D / P^{n}$ with $P$ a prime nonzero ideal and $n$ agreeing with the length of $D / P^{n}$. As a consequence, these $D$-modules are QI. Moreover the $D$-module $\left(D / P^{n}\right) \oplus\left(D / Q^{n}\right)$ with $P \neq Q$, is also QI hence $D / P^{n}$ and $D / Q^{n}$ are relatively injective [1, Proposition 2.2, p. 15]. Then applying Corollary 2.2, the class of the indecomposable $D$-modules of the same finite length is socle fine and the corollary is proved.

Let $D$ be a Dedekind domain and $T$ a nonzero torsion $D$-module. Then $T$ has a direct summand isomorphic either to $E(D / P)$ or to $D / P^{n_{P}}$ for some maximal ideal $P$ of $D$ and $n_{P} \in \mathbb{N}-\{0\}$ (see [18]). By using this, it can be proved that $M$ is a QP (quasi-projective) $D$-module if and only if $M$ is either a projective module or a torsion $D$-module such that for each maximal ideal $P$, its $P$-primary component is a direct sum of modules all isomorphic to $R / P^{n_{p}}$ for some $n_{p} \in \mathbb{N}-\{0\}$. It is easy to 
prove (see [1, Exercise 18, p. 24]) that if $D$ is a Dedekind domain and $M$ a finitely generated torsion $D$-module then $M$ is QI, if and only if $M$ is QP, if and only if $M \cong\left(D / P_{1}^{n_{1}}\right)^{m_{1}} \oplus\left(D / P_{2}^{n_{2}}\right)^{m_{2}} \oplus \cdots \oplus\left(D / P_{k}^{n_{k}}\right)^{m_{k}}$, where $P_{1}, P_{2}, \ldots, P_{k}$ are different maximal ideals of $D$, and $m_{i}, n_{i} \in \mathbb{N}-\{0\}$ for each $i=1,2, \ldots, k$.

Proposition 2.1. Let $D$ be a Dedekind domain. If $\mathcal{C}$ is a class of $Q P$ $D$-modules with nonzero socle and all of them with isomorphic radical, then $\mathcal{C}$ is socle fine. In particular any class of QI finitely generated torsion modules with isomorphic radicals is socle fine.

Proof: We consider $M$ and $N$ two elements of the class $\mathcal{C}$ with isomorphic socles. By the previous paragraph

$$
\begin{aligned}
& M \cong \bigoplus_{i \in I}\left(D / P_{i}^{m_{i}}\right)^{\left(A_{i}\right)}, \\
& N \cong \bigoplus_{j \in J}\left(D / Q_{j}^{n_{j}}\right)^{\left(B_{j}\right)}
\end{aligned}
$$

where $P_{i}$ and $Q_{j}$ are maximal, and $A_{i}, B_{j}$ are nonempty sets. By the fact that the socles are isomorphic we have the existence of a bijection $\sigma: I \rightarrow J$ such that $\left|A_{i}\right|=\left|B_{\sigma(i)}\right|, P_{i}=Q_{\sigma(i)}$ for all $i \in I$. On the other hand, after a suitable reordering, we can write $N \cong \bigoplus_{i \in I}\left(D / P_{i}^{n_{i}}\right)^{\left(A_{i}\right)}$ therefore

$$
\begin{aligned}
& \operatorname{Rad}(M) \cong \bigoplus_{i \in I}\left(D / P_{i}^{m_{i}-1}\right)^{\left(A_{i}\right)} \\
& \operatorname{Rad}(N) \cong \bigoplus_{i \in I}\left(D / P_{i}^{n_{i}-1}\right)^{\left(A_{i}\right)}
\end{aligned}
$$

and as their radicals are isomorphic their primary components are isomorphic also. Thus $\left(D / P_{i}^{m_{i}-1}\right)^{\left(A_{i}\right)} \cong\left(D / P_{i}^{n_{i}-1}\right)^{\left(A_{i}\right)}$. Futhermore their annihilators agree, that is to say, $P_{i}^{m_{i}-1}=P_{i}^{n_{i}-1}$ impliying $m_{i}=n_{i}$ for all $i$.

\section{Rings whose class of projective modules is socle fine}

It has been mentioned in the introduction, that the rings whose class of injective modules is socle fine are precisely the semiartinian rings. It is therefore natural to pose the question on the rings with socle fine class of projective modules. One first approach to the problem is given by the next theorem. 
Theorem 3.1. Let $R$ be a ring and $\mathcal{F}$ the class of the free $R$-modules. Then the following assertions are equivalent:

1) $\mathcal{F}$ is socle fine.

2) $R$ is an IBN ring with $\operatorname{Soc}(R) \neq 0$ and some homogeneous component in $\operatorname{Soc}(R)$ has finite length.

Proof: Suppose first that $\mathcal{F}$ is socle fine. If $\operatorname{Soc}(R)=0$ or no component has a finite length one checks immediately that $\operatorname{Soc}(R) \cong \operatorname{Soc}\left(R^{\mathbb{N}}\right)$ which take us to the contradiction $R \cong R^{\mathbb{N}}$. Next we prove that $R$ is IBN. If $R^{n} \cong R^{m}$ for $n, m \in \mathbb{N}$ then $\operatorname{Soc}\left(R^{n}\right) \cong \operatorname{Soc}\left(R^{m}\right)$. Since $\operatorname{Soc}(R)=$ $S_{i_{0}}^{k_{0}} \oplus\left(\oplus_{i \neq i_{0}} S_{i}^{\left(I_{i}\right)}\right)$ with $k_{0} \in \mathbb{N}^{*}$, and $S_{i_{0}}, S_{i}$ homogeneous components, this gives $S_{i_{0}}^{k_{0} n} \oplus\left(\oplus_{i \neq i_{0}} S_{i}^{\left(J_{i}\right)}\right) \cong S_{i_{0}}^{k_{0} m} \oplus\left(\oplus_{i \neq i_{0}} S_{i}^{\left(H_{i}\right)}\right)$ whence $k_{0} n=$ $k_{0} m$ and so $n=m$, as required. Let us prove 2) $\Rightarrow 1$ ). Consider two free modules $R^{(I)}, R^{(J)}$ with isomorphic socles and $\operatorname{Soc}(R)=S_{i_{0}}^{k_{0}} \oplus$ $\left(\oplus_{i \neq i_{0}} S_{i}^{\left(I_{i}\right)}\right)$ with $k_{0} \in \mathbb{N}^{*}$, and $S_{i_{0}}, S_{i}$ the homogeneous components. Then:

$$
\begin{aligned}
& \operatorname{Soc}\left(R^{(I)}\right)=S_{i_{0}}^{(X)} \oplus\left(\oplus_{i \neq i_{0}} S_{i}^{\left(I \times I_{i}\right)}\right) \\
& \operatorname{Soc}\left(R^{(J)}\right)=S_{i_{0}}^{(Y)} \oplus\left(\oplus_{i \neq i_{0}} S_{i}^{\left(J \times I_{i}\right)}\right)
\end{aligned}
$$

where $X=\left\{1, \ldots, k_{0}\right\} \times I, Y=\left\{1, \ldots, k_{0}\right\} \times J$. From the hypothesis that the socles are isomorphic one gets $|X|=|Y|$ hence $|I|=|J|$.

We recall that a ring $A$ is left pseudo-Frobenius (a left $\mathrm{PF}$ ring) if $A$ is an injective cogenerator. Left PF rings are characterized by the next theorem:

Theorem 3.2. Let $A$ be a ring. Then the following statements are equivalent:

1) $A$ is a left $P F$ ring.

2) The class of projective A-modules is socle fine and $A$ is a left cogenerator.

Proof: If $A$ is a left PF ring then by definition and $[3]$ and $[4]$ the second assertion holds. Suppose now that $A$ is a left cogenerator with its class of projective modules being socle fine. Then $\operatorname{Soc}(A) \neq 0$ since if $\operatorname{Soc}(A)=$ $0=\operatorname{Soc}(0)$ then $A=0$. Thus $\operatorname{Soc}(A)=\oplus_{i \in I} S_{i}=\oplus_{i \in I} \operatorname{Soc}\left(E\left(S_{i}\right)\right)$. As $A$ is a cogenerator then each $E\left(S_{i}\right)$ embeds in $A$ and $E\left(S_{i}\right)$ is a direct factor of $A$. Hence $E\left(S_{i}\right)$ is projective. We have then that $\oplus_{i \in I} E\left(S_{i}\right)$ is a projective $A$-module. Then since $\operatorname{Soc}(A)=\operatorname{Soc}\left(\oplus_{i \in I} E\left(S_{i}\right)\right)$ we get $A \cong \oplus_{i \in I} E\left(S_{i}\right)$. As $A$ is of finite type $A \cong \oplus_{i=1}^{n} E\left(S_{i}\right)$ for some $n \in \mathbb{N}$, whence $A$ is injective and as a consequence $A$ is a left $\mathrm{PF}$ ring. 
Theorem 3.3. Let $A$ be a ring, then the following properties are equivalent:

1) $A$ is a $Q F$ ring.

2) The class $\mathcal{D}$ of projective or injective modules is socle fine.

Proof: If $A$ is $\mathrm{QF}$, the class $\mathcal{D}$ is just the class of injective modules (and also the class of projective ones by [19, Theorem 13.6.1, p. 352]). Since $A$ is artinian, this class is socle fine by [16, Theorem 2]. Next we prove that if $\mathcal{D}$ is socle fine, then $A$ is a $\mathrm{QF}$ ring. Take $P$ a projective module, then we have $\operatorname{Soc}(P)=\operatorname{Soc}(E(P))$ and $P, E(P) \in \mathcal{D}$. Consequently $P \cong E(P)$ and so $P$ is injective. By [19, Theorem 13.6.1, p. 352], $A$ is QF.

Proposition 3.1. Let $A$ be a cogenerator ring. Then the following assertions are equivalent:

1) $A$ is a left QF3 ring.

2) The class of projective A-modules is socle fine.

Proof: If $A$ is a cogenerator left QF3 ring then $A$ is a PF-ring by [25, p. 55]. Now by Theorem 3.2 the class of projective $A$-modules is socle fine. The other implication is trivial applying Theorem 3.2.

Proposition 3.2. Let $A$ be a left QF3 ring. Then the following assertions are equivalent:

1) $A$ is a $Q F$-ring.

2) The class of projective A-modules is socle fine.

Proof: If $A$ is a QF-ring then the class of projective $A$-modules agrees with the class of injective modules and as $A$ is an artinian ring, this class is socle fine (see [16, Theorem 2]). Suppose that the class of projective $A$-modules is socle fine. Let $P$ be a projective $A$-module and $E(P)$ its injective hull. By $[8$, Corollary II.6, p. 58], both $P$ and $E(P)$ are projective. Since $\operatorname{Soc}(P)=\operatorname{Soc}(E(P))$ this implies $P \cong E(P)$ whence $P$ is injective and $A$ is a QF-ring.

\section{Semiperfect rings}

Let $A$ be a ring and $J$ its Jacobson radical. We recall that $A$ is semiperfect if $A / J$ is semisimple and any idempotent of $A / J$ is of the form $e+J$ with $e$ an idempotent of $A$. It is well known (see for instance [2, Proposition 27.10, p. 306]) that if $A$ is semiperfect, each complete family of primitive idempotents contains a basic family $e_{1}, \ldots, e_{m}$ of $A$, and all the basic families of $A$ have the same cardinality which is called the $c a$ pacity of $A$. The $A$-modules $A e_{1}, \ldots, A e_{m}$ form a complete irredundant 
family of representatives of projective indecomposable $A$-modules. The $A$-modules $A e_{1} / J e_{1}, \ldots, A e_{m} / J e_{m}$ form a complete irredundant family of representatives of simple $A$-modules. We shall use the notation $S_{i}=$ $A e_{i} / J e_{i}$ for all $i$. If $P$ is a projective $A$-module, then $P$ has an essentially unique decomposition of the type $P=\left(A e_{i}\right)^{\left(I_{1}\right)} \oplus \cdots \oplus\left(A e_{m}\right)^{\left(I_{m}\right)}$. A ring $A$ is called finitely embedded if and only if it has an essential and finitely generated socle. Any artinian ring is finitely embedded but there are rings which are finitely embedded (and even local) but nonartinian (see $[\mathbf{2 4}]$ ).

Theorem 4.1. Let $A$ be a finitely embedded semiperfect ring. The following assertions are equivalent:

1) The class of projective A-modules is socle fine.

2) A contains all its types of simple A-modules, and any projective indecomposable module has a homogeneous socle.

Proof: Consider a finitely embedded semiperfect ring $A$ with capacity $m$ and a basic family $e_{1}, \ldots, e_{m}$ of $A$. Suppose that the class of projective $A$-modules is socle fine. If $m=1$ then assertion 2) follows from the fact that $\operatorname{Soc}(A) \neq 0$. Next we take $m \geq 2$, and suppose (after a suitable reordering if necessary) that $\operatorname{Soc}(A) \cong S_{1}^{\alpha_{1}} \oplus \cdots \oplus S_{r}^{\alpha_{r}}$ with $\alpha_{i} \neq 0$ for $i \in\{1, \ldots, r\}$, and $r<m$. Then there is a $t \leq r$ such that $S_{1} \oplus \cdots \oplus S_{r}$ can be embedded in $A e_{i_{1}} \oplus \cdots \oplus A e_{i_{t}}$. Consequently $\operatorname{Soc}\left(A e_{i_{1}} \oplus \cdots \oplus\right.$ $\left.A e_{i_{t}}\right)=S_{1}^{\beta_{1}} \oplus \cdots \oplus S_{r}^{\beta_{r}}$ with $\beta_{i} \neq 0$ for all $i \in\{1, \ldots, r\}$. The projective $A$-modules $A^{(\mathbb{N})}$ and $\left(A e_{i_{1}} \oplus \cdots \oplus A e_{i_{t}}\right)^{(\mathbb{N})}$ have isomorphic socles hence we have an isomorphism

$$
A e_{1}^{(\mathbb{N})} \oplus \cdots \oplus A e_{m}^{(\mathbb{N})} \cong A e_{i_{1}}^{(\mathbb{N})} \oplus \cdots \oplus A e_{i_{t}}^{(\mathbb{N})} .
$$

Since $t<m$ there is a $j \in\{1, \ldots, m\}$ such that $j \notin\left\{i_{1}, \ldots, i_{t}\right\}$, but on the other hand, the theorem on the uniqueness of the decomposition of projective $A$-modules implies that $A e_{j} \cong A e_{i_{k}}$ for some $i_{k} \in\left\{i_{1}, \ldots, i_{t}\right\}$ which is contradictory. Next we prove that $\operatorname{Soc}\left(A e_{i}\right)$ is homogeneous for each $i$. Suppose that $\operatorname{Soc}\left(A e_{k}\right)=S_{1}^{\alpha_{1}} \oplus \cdots \oplus S_{q}^{\alpha_{q}}$ with $q \geq 2$ and $\alpha_{i} \neq 0$ for all $i$. Then there exist $A e_{i_{1}}, \ldots, A e_{i_{n}}$ pairwise different and distinct from $A e_{k}$ such that $n \leq m-q$, and $S_{q+1} \oplus \cdots \oplus S_{m}$ can be embedded in $A e_{i_{1}} \oplus \cdots \oplus A e_{i_{n}}$. Consequently $\operatorname{Soc}\left(A e_{k} \oplus A e_{i_{1}} \oplus \cdots \oplus A e_{i_{n}}\right)=$ $S_{1}^{\beta_{1}} \oplus \cdots \oplus S_{m}^{\beta_{m}}$ with each $\beta_{i} \neq 0$. As $\left(A e_{k} \oplus A e_{i_{1}} \oplus \cdots \oplus A e_{i_{n}}\right)^{(\mathbb{N})}$ is projective and its socle is isomorphic to the socle of the projective $A$-module $A^{(\mathbb{N})}$ we have an isomorphism

$$
A e_{k}^{(\mathbb{N})} \oplus A e_{i_{1}}^{(\mathbb{N})} \oplus \cdots \oplus A e_{i_{n}}^{(\mathbb{N})} \cong A e_{1}^{(\mathbb{N})} \oplus \cdots \oplus A e_{m}^{(\mathbb{N})}
$$


which implies that $n+1=m$ by the previously mentioned uniqueness theorem. Since we had $n \leq m-q$ and $q \geq 2$, then $n+1 \leq m-q+1 \leq$ $m-1$, a contradiction. Suppose now that 2 ) holds, and denote by $A e_{\sigma(i)}$ the unique direct summand of $A$ containing to $S_{i}$. It is clear that $i \mapsto \sigma(i)$ is a permutation of $\{1, \ldots, m\}$ and $\operatorname{Soc}\left(A e_{\sigma(i)}\right) \cong S_{i}^{n_{i}}$ with $n_{i} \neq 0$. Take $P$ and $Q$ two projective $A$-modules with $P=\oplus_{j=1}^{m} A e_{\sigma(j)}^{\left(I_{j}\right)}$ and $Q=\oplus_{j=1}^{m} A e_{\sigma(j)}^{\left(H_{j}\right)}$. If $\operatorname{Soc}(P) \cong \operatorname{Soc}(Q)$ we have that $\oplus_{j}\left(S_{j}^{n_{j}}\right)^{\left(I_{j}\right)} \cong$ $\oplus_{j}\left(S_{j}^{n_{j}}\right)^{\left(H_{j}\right)}$ and according to the uniqueness of the homogeneous components of semisimple modules we have $\left(S_{j}^{n_{j}}\right)^{\left(I_{j}\right)} \cong\left(S_{j}^{n_{j}}\right)^{\left(H_{j}\right)}$ for all $j$. The uniqueness of the decomposition of a semisimple module as a direct sum of simple ones implies the coincidence of cardinals: $\left|I_{j}\right|=\left|H_{j}\right|$ whence $P \cong Q$.

Corollary 4.1. Let $A$ be ring, of some of the following types:

1) A finitely embedded local ring.

2) A primary ring (that is $A$ is artinian and $A / \operatorname{Rad}(A)$ is simple).

3) A commutative artinian ring.

Then the class of projective A-modules is socle fine.

Proof: Suppose that $A$ is as in the first possibility. Since any local ring is semiperfect and all the simple $A$-modules are isomorphic, from $\operatorname{Soc}(A) \subset A$ we conclude that $A$ contains its unique type of simple $A$-module. As any projective $A$-module is free and $\operatorname{Soc}(A)$ is homogeneous we have that any projective indecomposable module has a homogeneous socle. In the second case, take into account that the simple modules over a simple artinian ring are isomorphic. It is easy to prove that the simple $A$-modules of the form $A e_{i} / \operatorname{Rad}(A) e_{i}$ are also simple as $A / \operatorname{Rad}(A)$-modules. Thus they are isomorphic as $A / \operatorname{Rad}(A)$-modules and also as $A$-modules. In this way there is only one isomorphism class of simple $A$-modules and the conditions in item 2 of Theorem 4.1 are satisfied. Finally, if $A$ is commutative and artinian, it splits into a finite direct sum of local artinian rings. The class of projective modules of any of these summands is socle fine (as proved in the first item), and from this it is easy to derive that the class of projective $A$-modules is socle fine.

In the noncommutative case we do not have in general this property. Take for instance a field $K$ and $A$ the artinian ring of triangular matrices defined by:

$$
A=\left(\begin{array}{cc}
K & 0 \\
K & K
\end{array}\right)
$$


Let $\mathrm{e}_{1}=\left(\begin{array}{ll}1 & 0 \\ 0 & 0\end{array}\right)$ and $\mathrm{e}_{2}=\left(\begin{array}{ll}0 & 0 \\ 0 & 1\end{array}\right)$. We have $A=A e_{1} \oplus A e_{2}$ and for all $i \in\{1,2\}$ the ring $e_{i} \mathrm{Ae}_{i} \simeq K$ is local. $A e_{1}$ and $A e_{2}$ are two projective $A$-modules with $\operatorname{Soc} A e_{1} \simeq \operatorname{Soc} A e_{2}$ but $A e_{1}$ and $A e_{2}$ are not isomorphic.

We recall that a ring $A$ is a left (resp. right) CEP-ring if any cyclic left (resp. right) $A$-module embeds essentially in a projective $A$-module. The QF and the uniserial rings are CEP-rings. Jain and LópezPermouth [15] have proved that if $A$ is a semiperfect CEP-ring, then the class of projective $A$-modules projectifs is socle fine. Any semiperfect CEP-ring is artinian. There is a finitely embedded semiperfect ring whose class of projective $A$-modules is socle fine and which is not a CEPring: let $K\left[x_{1}, x_{2}, \ldots\right]$ the ring of polynomials in an infinite countable number of commuting indeterminates $x_{1}, x_{2}, \ldots$, with entries in $K$. Let $I$ be the ideal of $K\left[x_{1}, x_{2}, \ldots\right]$ generated by $\left\{x_{i} x_{j} x_{k}, x_{n} x_{n+1}-x_{1} x_{2}, x_{l} x_{m}\right\}$ where $i, j, k, l, m$ and $n$ are positive integers with $|l-m| \neq 1$. Then the ring $A=\frac{K\left[x_{1}, x_{2}, \ldots\right]}{I}$ is a finitely embedded semiperfect ring which is not a CEP-ring and its class of projective $A$-modules is socle fine.

Acknowledgements. The authors would like to express their gratitude to the referee for his exhaustive work in corrections and suggestions improving notably the original version of the work.

\section{References}

[1] T. Albu And C. NĂStĂSESCU, "Relative finiteness in module theory', Monographs and Textbooks in Pure and Applied Mathematics 84, Marcel Dekker, Inc., New York, 1984.

[2] F. W. Anderson And K. R. Fuller, "Rings and categories of modules", Graduate Texts in Mathematics 13, Springer-Verlag, New York-Heidelberg, 1974.

[3] H. BASs, Finitistic dimension and a homological generalization of semi-primary rings, Trans. Amer. Math. Soc. 95 (1960), 466-488.

[4] J.-Y. Chamard, Anneaux semi-parfaits et presque-frobeniusiens, C. R. Acad. Sci. Paris Sér. A-B 269 (1969), A556-A559.

[5] Y. A. Drozd And V. V. KirichenKo, "Finite-dimensional algebras", Translated from the 1980 Russian original and with an appendix by Vlastimil Dlab, Springer-Verlag, Berlin, 1994.

[6] C. Faith, "Algebra. II. Ring theory", Grundlehren der Mathematischen Wissenschaften 191, Springer-Verlag, Berlin-New York, 1976.

[7] J. S. Golan, Characterization of rings using quasiprojective modules, Israel J. Math. 8 (1970), 34-38.

[8] J.-M. Goursaud, Anneaux unisérielles et unisérielles généralisés, These, Université de Poitiers (1970). 
[9] M. Harada, Note on quasi-injective modules, Osaka J. Math. 2 (1965), 351-356.

[10] A. Idelhadj, Classification d'anneaux par des proprietes relatives au socle et au radical de leurs modules, These, Faculté des sciences de Rabat, Université Mohammed V (1995).

[11] A. Idelhadj And E. A. KAIDI, A characterization of semiArtinian rings, in: "Commutative ring theory" (Fès, 1992), Lecture Notes in Pure and Appl. Math. 153, Dekker, New York, 1994, pp. 171-179.

[12] A. IDELhadJ AND E. A. KAIDI, Nouvelles caractérisations des $V$-anneaux et des anneaux pseudo-frobeniusiens, Comm. Algebra 23(14) (1995), 5329-5338.

[13] A. Idelhadj, E. A. Kaidi, D. Martín Barquero and C. MarTín GonzÁlez, Socle fine classes in Grothendieck categories, Algebras Groups Geom. 16(3) (1999), 401-409.

[14] N. Jacobson, "Structure of rings", American Mathematical Society, Colloquium Publications 37, American Mathematical Society, Prov., R. I., 1956.

[15] S. K. Jain And S. R. López-Permouth, Rings whose cyclics are essentially embeddable in projective modules, J. Algebra 128(1) (1990), 257-269.

[16] E. A. Kaidi, D. Martín Barquero and C. Martín González, Socle fine characterization of Artinian and Noetherian rings, Algebras Groups Geom. 10(3) (1993), 191-197.

[17] E. A. Kaidi, D. Martín Barquero and C. Martín GonzÁlez, On the socle-fine notion and modules of finite length, in: "Commutative ring theory" (Fès, 1995), Lecture Notes in Pure and Appl. Math. 185, Dekker, New York, 1997, pp. 369-375.

[18] I. Kaplansky, Modules over Dedekind rings and valuation rings, Trans. Amer. Math. Soc. 72 (1952), 327-340.

[19] F. KAsch, "Modules and rings", Translated from the German and with a preface by D. A. R. Wallace, London Mathematical Society Monographs 17, Academic Press, Inc. [Harcourt Brace Jovanovich, Publishers], London-New York, 1982.

[20] A. Koenler, Quasi-projective covers and direct sums, Proc. Amer. Math. Soc. 24 (1970), 655-658.

[21] D. Martín BArquero, Socle fine module classes for Dedekind domains, Journal de Mathematiques du Maroc 1, Publications de la Societe Mathématique du Maroc (1993), 48-53. 
[22] S. H. Mohamed and B. J. Müller, "Continuous and discrete modules", London Mathematical Society Lecture Note Series 147, Cambridge University Press, Cambridge, 1990.

[23] S. S. Page and Y. Q. Zhou, Direct sums of quasi-injective modules, injective covers, and natural classes, Comm. Algebra 22(8) (1994), 2911-2923.

[24] S. T. Rizvi, Commutative rings for which every continuous module is quasi-injective, Arch. Math. (Basel) 50(5) (1988), 435-442.

[25] E. A. Rutter, JR., PF and QF-3 rings, Arch. Math. (Basel) 19 (1968), 608-610 (1969).

[26] D. W. Sharpe and P. VÁmos, "Injective modules", Cambridge Tracts in Mathematics and Mathematical Physics 62, Cambridge University Press, London-New York, 1972.

\author{
Abdelouahab Idelhadj: \\ Département de Mathématiques \\ Université de Tétouan (Abdel Malek Essaadi) \\ 300 Tétouan \\ Morocco \\ El Amin Kaidi: \\ Departamento de Álgebra y Análisis Matemático \\ Facultad de Ciencias Experimentales \\ Universidad de Almería \\ 04120 Almería \\ Spain \\ E-mail address: elamin@ual.es \\ Dolores Martín Barquero: \\ Departamento de Matemática Aplicada \\ Universidad de Málaga \\ 29071 Málaga \\ Spain \\ E-mail address: dmartin@uma.es \\ Cándido Martín González: \\ Departamento de Álgebra, Geometría y Topología \\ Universidad de Málaga \\ 29080 Málaga \\ Spain \\ E-mail address: candido@apncs.cie.uma.es
}

Primera versió rebuda el 13 de novembre de 2003, darrera versió rebuda el 29 de març de 2004 